Wilfried Hendricks (Hrsg.)

Lernen mit Neuen Medien im Strafvollzug 
Reihe Pädagogik

Band 26 
Wilfried Hendricks (Hrsg.)

\section{Lernen mit Neuen Medien im Strafvollzug}

Evaluationsergebnisse aus dem Projekt e-LiS

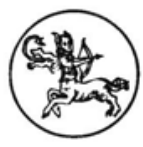

Centaurus Verlag \& Media UG 2005 


\section{Die Deutsche Bibliothek - CIP-Einheitsaufnahme}

Bibliographische Information der Deutschen Bibliothek:

Die Deutsche Bibliothek verzeichnet diese Publikation in der

Deutschen Nationalbibliographie; detaillierte bibliographische Daten

sind im Internet über http://dnb.ddb.de abrufbar

ISBN 978-3-8255-0549-3 ISBN 978-3-86226-433-9 (eBook)

DOI 10.1007/978-3-86226-433-9

\section{ISSN 0930-9462}

Alle Recbte, insbesondere das Recht der Vervielfältigung und Verbreitung sowie der Übersetzung, vorbebalten. Kein Teil des Werkes darf in ingendeiner Form (durch Fotokopie, Mikrofilm oder ein anderes Verfabren) obne schriftliche Genehmigung des V erlages reproduziert oder unter Verwendung elektronischer Systeme verareitet, vervielfältigt oder verbreitet werden.

(C) CENTAURUS Verlags-GmbH. \& Co. KG, Herbolzheim 2005

Satz: Vorlage des Herausgebers

Umschlaggestaltung: Daniela Nicolai 


\section{Inhaltsverzeichnis}

Vorwort

Das Projekt e-LiS in der Gemeinschaftsinitiative EQUAL .................................. 9

Telfi - das Partnerprojekt in Österreich ................................................................ 11

Aufgabenfelder der Entwicklungspartnerschaft e-LiS............................................. 11

Rolle und Aufgabe der Evaluation ......................................................................... 14

Vorschau auf die Beiträge des Buches .................................................................. 14

1 E-Learning - Einsatzformen und Erfolgsfaktoren

Sonja Förg ................................................................................................ 17

Einleitung ........................................................................................................ 17

Formen des E-Learnings .................................................................................... 18

Chancen und Risiken......................................................................................... 22

Voraussetzungen für effektives E-Learning und

die Rolle der Selbststeuerung ................................................................................. 23

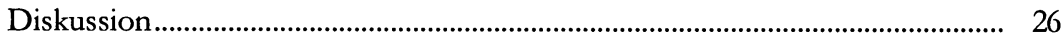

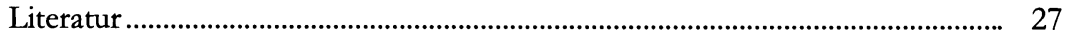

2 Der Computer kann Motivation und Leistung steigern

Karoline Schnetter ..................................................................................... $\quad 29$

Die Fragestellungen der Evaluation ................................................................... 29

Wer wurde befragt?............................................................................................... 30

Der Computer als wichtiger Anreizfaktor........................................................... 32

Computerkenntnisse sind nützlich ........................................................................ 33

Lernen am Computer motiviert ............................................................................. 34

Der Computereinsatz eignet sich für die Bildungseinrichtungen

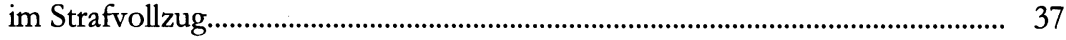

Nachhaltige Lernbereitschaft - Empowerment ................................................... 38

Computer-Einsatz als Erfolgsfaktor ..................................................................... 39

Fazit...................................................................................................... 42

Literatur .................................................................................................... 43 
3 Erfahrungen mit EDV-Kursen in der Justizvollzugsanstalt Bremen

Eduard Matt

Ausgangslage.

EDV-Kurse in der JVA Bremen....................................................................... 47

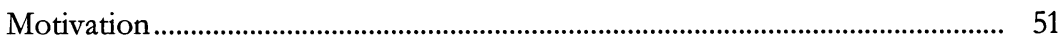

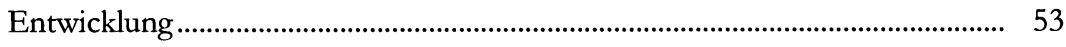

Stimmungsschwankungen........................................................................... 55

EDV und Arbeitsmarkt............................................................................... 56

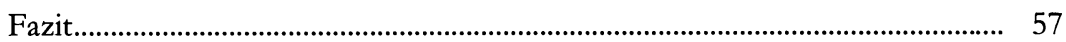

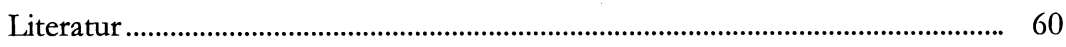

4 „Die Teilnahme hat sich auf jeden Fall gelohnt.“ - Ergebnisse der Begleitforschung zum Projekt TELFI

Claudia Sorger

Ausgangslage..................................................................................................... 63

Durchführung der Interviews ........................................................................... 65

Zugang zu den Kursen........................................................................................ 66

Räumlichkeiten und technische Ausstattung …………….................................. 66

Externe TrainerInnen.......................................................................................... 67

„Blended Learning“ in der Gruppe ...................................................................... 67

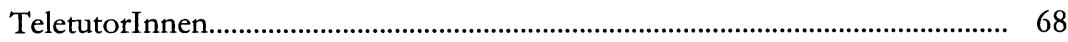

Psychologische Begleitung................................................................................. 69

Auswirkungen auf die Haftbedingungen ................................................................ 70

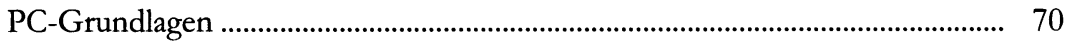

Deutsch als Fremdsprache............................................................................... 73

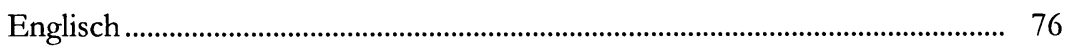

Lagerverwaltung ....................................................................................... 77

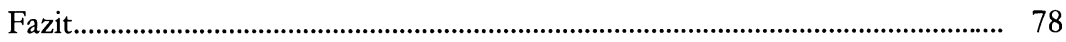

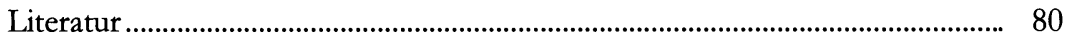


5 Computerkurse im Frauenstrafvollzug - ein Schlüssel zu neuen Lernerfahrungen und Berufsaussichten

Sonja Förg ................................................................................... 81

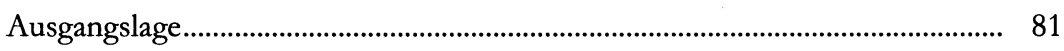

Durchführung und Auswertungsmethoden........................................................ 812

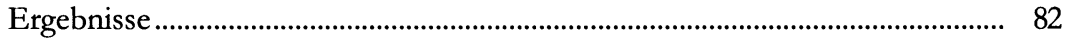

Zusammenfassung und Diskussion.............................................................. 94

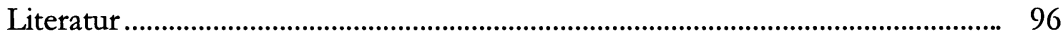

6 Formen der Betreuung beim selbstgesteuerten Lernen - Eine Fallstudie in der Jugendstrafanstalt Berlin

Birgit Lang - Karoline Schnetter.................................................... 97

Die Rahmenbedingungen .......................................................................... 97

Die Fragestellung der Fallstudie...................................................................... 100

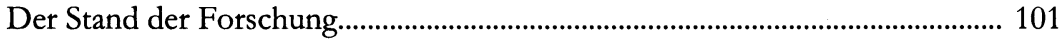

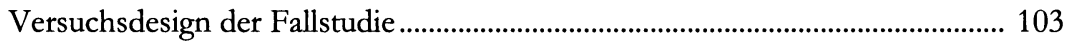

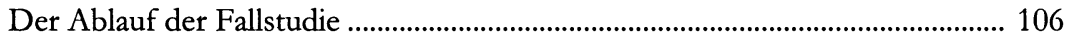

Die Ergebnisse der Fallstudie.......................................................................... 108

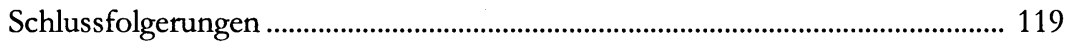

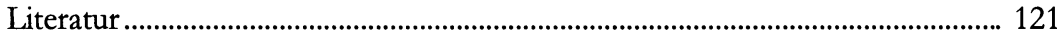

7 Lernsoftware im Unterricht - Eine Fallstudie in den

Jugendstrafanstalten Brandenburgs

Karoline Schnetter .................................................................................... 123

Das Brandenburger e-LiS Projekt im Aufgabenfeld Jugend (Teilprojekt 6) ..... 124

Die Befragung und Stichprobe ........................................................................ 124

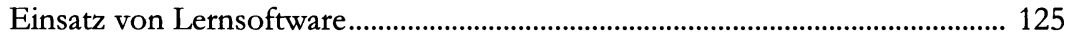

Vorteile und Hindernisse beim Einsatz von Lernsoftware aus Sicht der

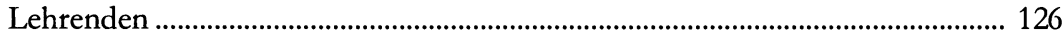

Fazit und Empfehlungen ................................................................................ 139

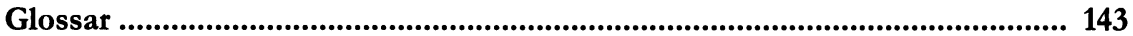

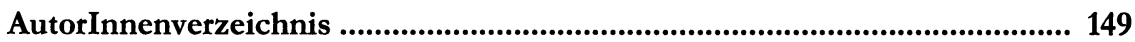




\section{Vorwort}

\section{Das Projekt e-LiS in der Gemeinschaftsinitiative EQUAL}

Das EU-Projekt e-LiS (e-Learning im Strafvollzug) bringt die sog. Neuen Medien ins Gefängnis. Es will dazu beitragen, die Beschäftigungschancen von inhaftierten Frauen, Männern und Jugendlichen auf dem Arbeitsmarkt zu verbessern, ihre Integration in die Gesellschaft zu erleichtern sowie eine erneute Straffälligkeit nach der Haftentlassung zu verhindern.

Neue Medien verbessern die Bildungsmöglichkeiten im Strafvollzug:

- Allgemein bildende und berufsbildende Angebote können mit dem Einsatz des Computers genauer auf die Bedürfnisse jedes Einzelnen zugeschnitten werden, was umso wichtiger erscheint, als in den Haftanstalten gerade Lernsondergruppen wie Lernbeeinträchtige und MigrantInnen überrepräsentiert sind.

- Da heutzutage der Umgang mit den Neuen Medien selbstverständlich geworden ist, sind die im Rahmen von e-LiS erworbenen Computerkenntnisse den Gefangenen bei der Arbeitssuche und im privaten Bereich nützlich.

- Durch die Integration der Neuen Medien in das Bildungsangebot der Haftanstalten erschließen sich neue Beschäftigungsfelder für die Inhaftierten, wie das Beispiel des Computerrecyclings zeigt.

Die in diesem Band vorgelegten Ergebnisse aus der Evaluation des Projektes e-LiS zeigen, dass die Integration Neuer Medien in die Bildungsangebote der beteiligen Haftanstalten weitgehend erfolgreich gelungen ist. Die positiven Lernerfahrungen der Inhaftierten tragen zu deren Beschäftigungsfähigkeit bei und leisten somit einen Beitrag dazu, den Teufelskreis von Inhaftierung, Ausgrenzung auf dem Arbeitsmarkt und erneuter Straffälligkeit zu durchbrechen.

e-LiS ist Teil der europäischen Gemeinschaftsinitiative EQUAL. Diese setzt sich auf breiter Basis für eine Verbesserung der Chancengleichheit, bessere Beschäftigungsmöglichkeiten und die Beseitigung von Diskriminierung auf dem Arbeitsmarkt ein. Europaweit werden in zwei Förderrunden Netzwerke von Projekten mit innovativen Ideen unterstützt. In der ersten Förderrunde von 2002 bis 2005 engagierten sich europaweit 1500 Projektverbünde, sog. Entwicklungspartnerschaften (EPs), für benachteiligte Zielgruppen auf dem Arbeitsmarkt. In Deutschland waren dabei 109 EPs mit 1100 Teilprojekten (TPs) beteiligt. Die EPs setzten sich jeweils aus EinzelakteurInnen 
einer Region oder eines Sektors zusammen, die aus verschiedenen Perspektiven gemeinsam an einem arbeitsmarktpolitischen Problem arbeiteten.

Das Projekt e-LiS war eine der größten deutschen Entwicklungspartnerschaften der ersten EQUAL-Förderrunde. Es wurde in sechs norddeutschen Bundesländern umgesetzt: Berlin, Brandenburg, Bremen, Hamburg, Mecklenburg-Vorpommern und Schleswig-Holstein. In allen Ländern waren die Justizverwaltungen sowohl die Initiatoren als auch aktiv an der Arbeit des Projektes beteiligt. e-LiS hat insgesamt 32 Teilprojekte ${ }^{1}$.

Die EP e-LiS arbeitete nach einer vorbereitenden Phase für drei Jahre vom 16. Mai 2002 bis zum 15. Mai 2005. Die Teilprojekte wurden von verschiedenen Bildungsträgern umgesetzt und widmeten sich jeweils verschiedenen Aspekten und Schwerpunkten des Themas E-Learning im Strafvollzug. So beschäftigten sich zum Beispiel einzelne Teilprojekte mit dem mediengestützten Profiling und Assessment von jugendlichen Strafgefangenen, mit E-Learning im Frauenstrafvollzug oder mit der Weiterbildung der Lehrkräfte. Kernstück der gemeinsamen Arbeit in e-LiS war eine Lernplattform, die an die speziellen Bedürfnisse des Strafvollzugs angepasst wurde. Sie ermöglicht die Bereitstellung von gemeinsamem Lernmaterial und ein lernortübergreifendes Lernen in den angeschlossenen Haftanstalten.

EQUAL-Projekte werden aus dem Europäischen Sozialfonds sowie nationalen Mitteln finanziert. Als eine Art Forschungs- und Entwicklungsprogramm des Europäischen Sozialfonds entwickeln sie innovative Ansätze der Arbeitsmarktpolitik mit unterschiedlichen thematischen Schwerpunkten. Gemeinsames Anliegen aller Entwicklungspartnerschaften ist das Ziel, erfolgreiche Initiativen nach der Erprobung in die Regelsysteme der Arbeitsmarktpolitik zu überführen. Dieser Prozess wird als Mainstreaming bezeichnet. Er richtet sich auf regionaler, nationaler und auch europäischer Ebene an die maßgeblichen Arbeitsmarktakteure wie die Politik, die Sozialpartner und Bildungseinrichtungen.

e-LiS hat die EQUAL-Strategie des Mainstreaming bereits weitgehend erfolgreich und nachhaltig umgesetzt:

- Sowohl die Kooperationsstrukturen zwischen den beteiligten Bundesländern als auch die aufgebaute Lernplattform werden nach Projektende weiter bestehen und ausgebaut.

- Die gewonnenen Erkenntnisse und Erfahrungen zum Einsatz Neuer Medien im Unterricht werden weiter für die Bildungsarbeit im Strafvollzug genutzt.

1 Siehe auch www.e-lis.de 
- Der Einsatz Neuer Medien im Strafvollzug wird - unter anderen Schwerpunkten in mehreren neuen Entwicklungspartnerschaften der zweiten Förderrunde von EQUAL weiterentwickelt.

Die Arbeit von Entwicklungspartnerschaften in der Gemeinschaftsinitiative EQUAL geht über den nationalen Rahmen hinaus. In vielfältigen transnationalen Aktivitäten wurden mit Entwicklungspartnerschaften anderer europäischer Staaten Konzepte, Erfahrungen und Ergebnisse ausgetauscht und gemeinsam Produkte entwickelt. e-LiS arbeitete hier u. a. mit Partnern aus Frankreich, Großbritannien, den Niederlanden, Österreich und Spanien zusammen.

\section{TELFI - das Partnerprojekt in Österreich}

Die österreichische Entwicklungspartnerschaft TELFI (Telelernen für HaftinsassInnen) versuchte ebenfalls, einen Beitrag dazu zu leisten, dass in Zukunft mehr Strafgefangene besser auf die Anforderungen des Lebens nach einer Haft, besonders unter den Perspektiven des Arbeitsmarktes, vorbereitet werden. Durch E-Learning-Kurse für HaftinsassInnen mit arbeitsmarktrelevanten Inhalten werden die im Strafvollzug bereits bestehenden Weiterbildungsangebote ergänzt.

Qualifikationsmaßnahmen alleine sind oft nicht ausreichend, um die Chance der Integration von Gefangenen oder Haftentlassenen in den Arbeitsmarkt wirklich zu verbessern. Dazu bedarf es einer integrierten Annäherung, die das „Davor“, das „Danach" und auch das "Jetzt" mit einbezieht. Im Rahmen von TELFI werden die KursteilnehmerInnen daher in einem von Psychologinnen geleiteten Auswahlverfahren ausgewählt und erhalten wöchentliche Gruppengespräche.

TELFI ist zwar auf die Projektlaufzeit vom 15. September 2002 bis zum 14. September 2005 sowie auf die sechs Projektanstalten Schwarzau, Gerasdorf, Wien-Simmering, Wien-Josefstadt, Stein und Wels beschränkt; die Angebote, Erfahrungen und Produkte des Projektes sollen aber auch nach der Projektzeit weiter genutzt werden. Nicht zuletzt im Hinblick auf die Etablierung und Ausweitung von E-Learning im Strafvollzug wird im Rahmen von TELFI ein eigener „Strafvollzugsbildungs-Server“ eingerichtet, der Organisation und Durchführung von Kursen unterstützen und erleichtern soll.

\section{Aufgabenfelder der Entwicklungspartnerschaft e-LiS}

Die EP e-LiS fasste ihre vielfältigen Aktivitäten in Aufgabenfeldern zusammen, in denen einzelne Teilprojekte miteinander themenspezifisch kooperierten. Zudem wur- 
den einzelne Querschnittsaufgaben, wie Gender Mainstreaming und Evaluation, von einzelnen Partnern für die gesamte EP wahrgenommen.

\section{Jugendliche}

Eines der Kernziele von e-LiS war die Verbesserung der Chancen von sozial benachteiligten bzw. delinquenten Jugendlichen beim Zugang zu Ausbildung und Erwerbstätigkeit. Im Jugendstrafvollzug gab es unterschiedliche Akzentsetzungen bei der Arbeit mit und am Computer, wie z. B. Hardwarekurse, Computerrecycling, Nutzung von Bildungssoftware im allgemein bildenden Unterricht sowie berufliche Orientierungsund Qualifizierungskurse zur Unterstützung bei der Haftentlassung. Die Entwicklung von Medienkompetenz hatte einen hohen Stellenwert in verschiedenen Teilprojekten.

\section{Frauen}

In den Justizvollzugsanstalten für Frauen lernten die Inhaftierten in den Bereichen der allgemeinen und beruflichen Bildung mit Softwareunterstützung. Sie entwickelten darüber hinaus ihre Medienkompetenz. Durch unterschiedliche Lernmodule und -aktivitäten, die aufeinander aufbauten, aber auch einzeln nutzbar waren, konnte auf die unterschiedlichen Bedürfnisse der inhaftierten Frauen eingegangen werden. So erwarben Haftinsassinnen auf diese Weise zum Beispiel den Europäischen Computerführerschein oder nutzten in ihrer Freizeit computerbasierte Lerninseln zur Weiterbildung.

\section{Männer}

Durch die neuen Technologien konnte e-LiS das schulische und berufliche Lernen von inhaftierten Männern modernisieren. Neben der Vermittlung von informationstechnischen Kenntnissen spielte vor allem der Erwerb der Fähigkeit, mit verfügbaren Informationen verantwortungsvoll umzugehen, eine wichtige Rolle. Es wurden mediengestützte Qualifizierungsmodule im Bereich der Grundbildung neu entwickelt und erprobt oder mit dem Computerrecycling ein neues Beschäftigungsfeld für die Gefangenen erschlossen.

\section{Train the Trainer}

e-LiS wollte die umfassende Anwendung multimedialen Lernens im Schul- und Berufsschulunterricht der Justizvollzugsanstalten erreichen. Die Lehrenden müssen dazu sowohl über die angemessene didaktisch-methodische Kompetenz im Umgang mit Informations- und Kommunikationstechnologien als auch über ein entsprechendes 
technisches Wissen verfügen. Den Lehrenden, die im Strafvollzug unterrichten, wurde deshalb eine Vielzahl entsprechender Fortbildungen angeboten. Außerdem fand für die MitarbeiterInnen der Anstalten Weiterbildung zu vollzuglichen Themen statt. Hierfür wurden multimediale Lerneinheiten, zum Beispiel zu Sicherheitsbelangen oder zur Gesundheitsförderung, in Haftanstalten produziert und auf der Lernplattform angeboten.

\section{Lernplattform}

e-LiS entwickelte eine eigene technologische Infrastruktur für webbasierte E-Learning-Konzepte. Die e-LiS-Lernplattform wurde auf der Basis einer OpenSource-Lösung (ILIAS) den speziellen Bedürfnissen der Bildungsarbeit im Strafvollzug angepasst. Von besonderer Bedeutung waren hierbei die Sicherheitsbedürfnisse des Strafvollzugs, die ein eigenes Sicherheitskonzept für die Lernplattform notwendig machten. Dieses ermöglicht es, auf der Lernplattform zentral Lerninhalte anzubieten, die von den beteiligten Haftanstalten über sichere Internet-Verbindungen abgerufen werden können. Die Lernplattform unterstützt zudem anstaltsübergreifende Lernortkooperationen und Weiterbildungsangebote für das Personal des Strafvollzugs ${ }^{2}$.

\section{Gender Mainstreaming}

Gender Mainstreaming steht für die Herstellung von Chancengleichheit beider Geschlechter auf allen Ebenen und in allen Maßnahmen von Entwicklungspartnerschaften. Es ist ein gemeinsames Ziel aller EQUAL-Entwicklungspartnerschaften. Insofern war auch e-LiS bestrebt, den Ansatz des Gender Mainstreaming in den beteiligten Institutionen und Arbeitsgebieten umzusetzen. In den einzelnen Teilprojekten wurden hierfür spezielle Beratungs- und Schulungsmaßnahmen angeboten.

\section{Evaluation}

Entwicklungspartnerschaften innerhalb von EQUAL werden extern evaluiert, $d . h$. deren Entwicklungsprozesse in den Teilprojekten werden kritisch begleitet und bewertet. Für die Entwicklungspartnerschaft e-LiS hat das IBI - Institut für Bildung in der Informationsgesellschaft (Berlin) die Evaluation übernommen. Es zeichnet auch für die vorliegende Veröffentlichung verantwortlich.

2 Der Einsatz der Lernplattform in Bildungsmaßnahmen Strafgefangener erfolgte am Ende des e-LiS-Projektes. Die Evaluation konnte aus Zeitgründen die Erfahrungen mit der Lernplattform nicht untersuchen. 


\section{Rolle und Aufgabe der Evaluation}

Welche Aufgaben hat die Evaluation im Kontext des EQUAL-Programms und der Entwicklungspartnerschaften zu erfüllen? Wir verstehen unter Evaluation die „systematische Anwendung sozialwissenschaftlicher Forschungsmethoden zur Beurteilung der Konzeption, Ausgestaltung, Umsetzung und des Nutzens sozialer Interventionsprogramme. "(Rossi, Freeman und Hofmann, 19883).

Die Programmevaluation von EQUAL ist für die Gesamtbewertung des Programms und der Programmerfolge in Deutschland zuständig. Daneben verfügt jede Entwicklungspartnerschaft in EQUAL jeweils über eine eigene Evaluation, die die Entwicklungsprozesse im Projekt kritisch begleitet und bewertet.

Den Schwerpunkt der Evaluation für e-LiS hat das IBI auf die Prozessbegleitung, d. h. auf die formative Evaluation gelegt. Die Ergebnisse und daraus abgeleitete Schlussfolgerungen und Empfehlungen wurden regelmäßig an die Beteiligten zurück gemeldet und gemeinsam diskutiert. Evaluation war somit als konstruktives Begleiten der Teilprojekte und der Entwicklungspartnerschaft gedacht. Mit einem multiperspektivischen Zugang stellte das IBI sicher, dass verschiedene Sichtweisen und Ebenen miteinbezogen werden konnten (also z. B. ProjektleiterInnen und Kurs-TeilnehmerInnen). Daneben wurden auch - im Sinne einer Erfolgskontrolle (summative Evaluation) die Stärken und Schwächen am Ende des Projekts analysiert. Außerdem hat die Evaluation die Aufgabe, die Projektergebnisse zu dokumentieren und einer breiteren Öffentlichkeit zugänglich zu machen.

Die Unterschiedlichkeit der Einzelprojekte innerhalb von e-LiS stellte eine große Herausforderung für die Evaluation dar. Um ein möglichst umfassendes Bild zu erhalten, wurden sehr viele Teilprojekte in eine Evaluation miteinbezogen, die teilprojektübergreifende Fragestellungen verfolgte; für die nötige Tiefe sorgten ergänzende Fallstudien, die in Absprache mit den ProjektleiterInnen entworfen und durchgeführt wurden.

\section{Vorschau auf die Beiträge des Buches}

Förg geht in ihrem einleitenden Beitrag „E-Learning - Einsatzformen und Erfolgsfaktoren“ auf die Entstehung des Begriffs „E-Learning“ sowie auf Formen und Voraussetzungen ein. Außerdem diskutiert sie Chancen und Grenzen von E-Learning.

3 Rossi, P. H., Freeman, H. E., Hofmann, G. (1988). Programm-Evaluation. Einführung in die Methoden angewandter Sozialforschung. Stuttgart: Enke. 
Schnetter zeigt in dem Beitrag „Der Computer kann Motivation und Leistung steigern" anhand der teilprojektübergreifenden Evaluationsergebnisse, inwieweit sich der Computer als Motivationswerkzeug für Strafgefangene eignet. Sie kommt zu dem Schluss, dass sowohl aus Sicht der TeilnehmerInnen als auch der DozentInnen EDVGrundlagen-Kurse und Unterricht mit Unterstützung des Computers sinnvolle Innovationen im Bildungsbereich des Strafvollzugs sind. Ferner weist sie darauf hin, dass der Unterricht durch den Einsatz von Computern effektiver wird: Die Leistungen der jugendlichen Strafgefangenen sind umso besser, je häufiger sie mit dem Computer lernen - unabhängig davon, ob sie den Umgang mit Microsoft Office lernen oder sich mit Lernsoftware weiterbilden.

Matt beleuchtet in dem Beitrag „Erfahrungen mit EDV-Kursen in der Justizvollzugsanstalt Bremen“ die Besonderheiten des Erwachsenenstrafvollzugs für Männer und berichtet über ausgesuchte Ergebnisse aus zwei Kursen in der JVA Bremen. Er legt dar, wie sich die Motivation und das Teilnahmeverhalten durch die Kurse verändern und welche Bedeutung die EDV-Kurse für die Arbeitsmarktintegration aus Sicht der Teilnehmer haben. Neben der Vermittlung von Fachkenntnissen steht der Aufbau von Selbstbewusstsein und sozialen Kompetenzen im Vordergrund, so sein Resümee.

Sorger berichtet in dem Beitrag „Die Teilnahme hat sich auf jeden Fall gelohnt - Ergebnisse der Begleitforschung zum Projekt TELFI“ von den Ergebnissen aus dem österreichischen Projekt TELFI - Telelernen für HaftinsassInnen. Sie geht kurz auf die Lage des österreichischen Strafvollzugs ein und beschreibt das Projekts TELFI. Ihren Ergebnissen zufolge werden die Kurse und die begleitenden Maßnahmen (psychologische Begleitung und Nachsorgearbeit) durchweg sehr positiv bewertet.

Förg referiert in dem Beitrag „Computerkurse im Frauenstrafvollzug - ein Schlüssel zu neuen Lernerfahrungen und Berufsaussichten" die Ergebnisse einer e-LiSFallstudie. Hier wurde die Wirkung von Computerkursen im Frauenstrafvollzug im Rahmen einer qualitativen Befragung untersucht. Sie kommt zu dem Schluss, dass das Lernen mit und am Computer neben dem Erwerb von Kompetenzen und Wissen von den Frauen im Strafvollzug als eine wichtige Grundlage für die berufliche und soziale Integration nach der Entlassung angesehen wird. Die Frauen gewinnen wieder Spaß am Lernen, sind motiviert und leistungsorientiert, erweitern ihr Berufsspektrum und haben positive berufliche Selbstwirksamkeitserwartungen. Nebenbei gibt es Anzeichen, dass das Lernen mit dem Computer den Erwerb von Schlüsselkompetenzen fördert.

Schnetter geht in ihrem Beitrag „Lernsoftware im Unterricht - eine Fallstudie in den Jugendstrafanstalten Brandenburgs" auf die Erfahrungen mit dem Einsatz von Bildungssoftware innerhalb von e-LiS an den Jugendstrafanstalten des Landes Brandenburg ein. Sie hat dazu telefonische Interviews mit zehn LehrerInnen durchgeführt. Sie 
kommt zu dem Ergebnis, dass die Lehrenden sehr unterschiedlich gegenüber Bildungssoftware eingestellt sind und dementsprechend unterschiedlich stark bereit sind, Software in ihren Unterricht zu integrieren. Neben Vorteilen, wie z. B. mehr Motivation seitens der Lernenden oder neue Möglichkeit der Binnendifferenzierung, sehen die Lehrenden Probleme, z. B. in der Technik und in der Qualität und der Quantität der Ausstattung. Schnetter sieht einen Zusammenhang zwischen Intensität der Erfahrung in der Arbeit mit (Bildungs-)Software und Belastungen durch Nutzung von Software: Je mehr Erfahrung, desto geringer die Probleme. Sie stellt dar, welche Kompetenzen Lehrende haben, die Lernsoftware regelmäßig einsetzen, und wie sie ihre Bildungsarbeit didaktisch-methodisch gestalten. Abschließend gibt sie Empfehlungen, wie der Einsatz von Lernsoftware im Unterricht weiter gefördert werden kann.

Schnetter und Lang berichten in ihrem Beitrag „Formen der Betreuung beim selbstgesteuerten Lernen“ über die Ergebnisse einer weiteren e-LiS-Fallstudie. In dieser experimentellen Studie untersuchten sie, wie intensiv die Betreuung während einer Schulung sein muss, um möglichst gute Lernergebnisse zu erhalten. Mittels statistischer Analysen bestätigen die Autorinnen ihre Hypothese, dass die Art des Kontaktes zwischen Auszubildendem und Lerncoach mit Motivation und Leistung der Lernenden zusammenhängt. Der Beitrag beschreibt daneben auch die Erfahrungen der Lerncoaches und der Auszubildenden, die zum Schluss führen, dass eine virtuelle Betreuung über einen Chat für die Zielgruppe nicht ausreichend ist. Abschließend werden verschiedene Empfehlungen erörtert, wie E-Learning in Zukunft im Strafvollzug effektiv eingesetzt werden kann.

Berlin im Mai 2005

Wilfried Hendricks - Christian Pfeffer-Hoffman 